\title{
Selección de variables relacionadas con fallos de chumaceras aplicando reconocimiento lógico combinatorio de patrones
}

\author{
Selection of variables related to journal bearing faults through \\ logical combinatorial pattern recognition
}

\author{
Joel Pino Gómez $^{1 *} \quad$ Fidel Ernesto Hernández Montero ${ }^{1} \quad$ Julio César Gómez Mancilla $^{2}$ \\ Recibido 03 de mayo de 2018, aceptado 23 de diciembre de 2018 \\ Received: May 03, 2018 Accepted: December 23, 2018
}

\begin{abstract}
RESUMEN
En el diagnóstico de fallos de chumaceras actualmente no se considera el conocimiento experto expresado en variables no numéricas, a pesar de constituir una fuente de información importante. Este trabajo se desarrolló con el objetivo de identificar los rasgos más relevantes para clasificar un grupo de fallos ocurridos en las chumaceras de una turbina de vapor. Las variables que soportan el trabajo corresponden a los datos almacenados en reportes de diagnóstico y mantenimiento de una termoeléctrica en explotación. Las técnicas aplicadas para procesar los datos cuantitativos y cualitativos son herramientas de reconocimiento lógico combinatorio de patrones (RLCP). Se determinó la confusión de los rasgos del conjunto inicial y posteriormente los testores y testores típicos, y se calculó el peso informacional de los rasgos. Los resultados alcanzados mostraron que la relevancia de los rasgos cualitativos presentes en la descripción de los fallos es superior a la de los rasgos numéricos típicamente empleados.
\end{abstract}

Palabras clave: Rasgos mezclados, selección, confusión, testores.

\section{ABSTRACT}

The text experts in industrial diagnostics can provide essential information, expressed in mixed variables (quantitative and qualitative), about journal bearing faults. However, researches on feature selection for fault diagnostic applications disobey the important knowhow expertise. This work is focused on the identification of the most important features for fault identification in a steam turbine journal bearings. The values sets that support this research come from stored diagnostics and maintenance reports from an active thermoelectric power plant. Mixed data processing was accomplished by means of logical combinatorial pattern recognition tools. Confusion of raw features set was obtained by employing different comparison criteria. Subsequently, the testor and typical testor were identified and the informational weight of features that conform typical testor was also computed. The high importance of the mixed features that came from the expert knowledge was revealed by the obtained achievements.

Keywords: Mixed features, features selection, logical combinatorial patter recognition, diagnostic, journal bearing.

1 Universidad Tecnológica de la Habana (CUJAE). Facultad de Ingeniería en Telecomunicaciones. La Habana, Cuba. E-mail: joelpinogomez@gmail.com; fhernandez@tele.cujae.edu.cu

2 Instituto Politécnico Nacional (IPN). Escuela Superior de Ingeniería Mecánica (ESIME). Ciudad de México, México. CP 07738. E-mail: gomezmancilla@gmail.com

* Autor de correspondencia: joelpinogomez@gmail.com 


\section{INTRODUCCIÓN}

Los mayores porcientos de las averías que ocurren en máquinas rotatorias están vinculados con los fallos de los rodamientos [1-3]. Gran parte de la industria que centra su funcionamiento en grandes máquinas rotatorias emplea chumaceras. Aumentar y mantener buenos índices de disponibilidad en importantes máquinas, así como mejorar el impacto de los trabajos de mantenimiento que se realicen, depende en gran medida del correcto funcionamiento y diagnóstico de las chumaceras [4]. $\mathrm{El}$ adecuado análisis de la estabilidad dinámica de las chumaceras debe basarse en la observación de una gran variedad de factores, algunos tangibles y otros no que son expresados en ocasiones mediante un lenguaje no formalizado matemáticamente por parte de los especialistas [5-8]. El conocimiento experto sobre los fallos que ocurren en las chumaceras de importantes máquinas rotatorias se expresa en variables de diferente naturaleza, es decir tanto en variables cuantitativas como cualitativas. Seleccionar qué variables se deben utilizar para el diagnóstico dado el conjunto completo de rasgos disponibles, tanto numéricos como no numéricos, es de vital importancia para alcanzar buenos resultados en la clasificación.

Hasta ahora los trabajos desarrollados para diagnosticar chumaceras utilizan solo variables cuantitativas que son procesadas utilizando redes neuronales artificiales (ANN), análisis discriminante de Fisher (FDA), máquinas de soporte vectorial (SVM) y K vecinos cercanos (KNN) [9-13], aspecto que limita la efectividad de las técnicas desarrolladas.

El RLCP aporta herramientas muy útiles para el trabajo con variables mezcladas (rasgos numéricos y no numéricos) que lo hacen idóneo para realizar la selección de los rasgos necesarios para describir los fallos en las chumaceras [14]. El trabajo que se presenta propone la aplicación del RLCP a la identificación de los rasgos más importantes que describen fallos en chumaceras de una turbina de vapor. Los conjuntos de valores de las variables que soportan el trabajo corresponden a los datos almacenados en reportes de diagnóstico y mantenimiento de una termoeléctrica cubana en explotación.

\section{ANÁLISIS TEÓRICO. RLCP}

El RLCP se nutre de la Lógica Matemática, la Teoría de Testores, la Teoría Clásica de Conjuntos, la Teoría de los Subconjuntos Difusos, la Teoría Combinatoria y la Matemática Discreta en general. Esencialmente los objetos son descritos por medio de una combinación de rasgos de diferente naturaleza de manera que todos (incluidos los cualitativos) pueden ser procesados por funciones numéricas. El primer problema que atiende el RLCP es la selección de variables, y seguidamente puede atender diferentes tipos de problemas de clasificación [14]. El problema que trata este trabajo es de selección de variables, lo cual consiste en encontrar los rasgos que inciden en el problema de manera determinante y reducir el número de rasgos en términos de los cuales se deben describir los objetos. El RLCP aporta criterios de comparación para valores de una variable útiles en primera instancia para desarrollar la selección de variables.

\section{Criterios de Comparación}

Un criterio de comparación consiste en una formulación matemática que permite, dados dos valores de un rasgo, calcular el nivel de semejanza o similaridad entre ellos. A continuación, se describen brevemente los criterios de comparación (CC) empleados en este trabajo [15].

El primer criterio de comparación abordado, el criterio de comparación de igualdad (CC1), se empleó con variables no numéricas debido a que el dominio de las variables numéricas utilizadas pertenece al conjunto de los números reales y en tal condición carece de sentido el empleo de este criterio. El CC1 establece que dos valores de un rasgo son semejantes siempre que dichos valores sean iguales, como se describe en la siguiente expresión:

$$
C C 1(X s(O i), X s(O j))=\left\{\begin{array}{l}
1 \text { si } X s(O i)=X s(O j) \\
0 \text { en otro caso }
\end{array}\right.
$$

donde $\mathrm{XsO}$ i y $\mathrm{XsO}$ j son los valores del rasgo s para los objetos $O i$ y $O j$, respectivamente.

Otro criterio de comparación empleado en el trabajo, el criterio de pertenencia a un intervalo (CC2), se describe a través de la siguiente expresión:

$$
C C 2(X s(O i), X s(O j))=\left\{\begin{array}{l}
1 \text { si } X s(O i), X s(O j) \in \mid a_{p}, a_{p+1} \\
0 \text { en otro caso }
\end{array}\right.
$$

El CC2 establece que, si dos valores de un rasgo están dados en un mismo intervalo, entonces son semejantes. De lo contrario, son diferentes. 
La semejanza entre dos datos aritméticos también puede determinarse como una función de las distancias entre ellos, el criterio de cercanía de datos aritméticos (CC3), expresa semejanza en caso de que la diferencia entre dos valores de un rasgo no exceda un umbral, tal como se muestra en la siguiente expresión [15]:

$$
C C 3(X s(O i), X s(O j))=\left\{\begin{array}{l}
1 \text { si }|X s(O i)-X s(O j)| \leq \in_{s} \\
0 \text { en otro caso }
\end{array}\right.
$$

Otro criterio de comparación utilizado en el trabajo es el criterio de cercanía normalizada de datos aritméticos (CC4), el cual también expresa proximidad aritmética entre dos valores de un rasgo. La semejanza de acuerdo a este criterio se define de acuerdo a la siguiente ecuación:

$$
C C 4(X s(O i), X s(O j))=1-\frac{|X s(O i)-X s(O j)|}{\Delta X s}
$$

En este caso la diferencia calculada se normaliza a la máxima diferencia admisible $(\Delta \mathrm{Xs})$ y la sustracción a 1 se realiza para expresar similaridad. A diferencia de los CC2 y CC3, donde la salida es booleana, la salida de CC4 es real. Un resultado cercano a 1 expresa un alto grado de semejanza, mientras que uno próximo a 0 expresa lo contrario.

Un criterio que puede utilizarse tanto en variables cuantitativas como cualitativas es el criterio de pertenencia a un conjunto $(\mathrm{CC} 2 \mathrm{c})$, pero al igual que $\mathrm{CC} 1$, en este trabajo solo fue empleado para comparar rasgos no numéricos. El criterio $\mathrm{CC} 2 \mathrm{c}$ se define como:

$$
C C 2 c(X s(O i), X s(O j))=\left\{\begin{array}{l}
1 \text { si } X s(O i), X s(O j) \in A_{p} \\
0 \text { en otro caso }
\end{array}\right.
$$

y establece la semejanza entre dos valores si ambos pertenecen a un mismo conjunto (o a la unión finita de varios conjuntos) de valores posibles para el rasgo que se analiza.

\section{Confusión}

La confusión (error) introducida por un rasgo (o grupo de rasgos) se define como la cantidad de pares semejantes, de acuerdo a algún criterio de comparación, de valores de un rasgo (o grupo de rasgos) que pertenecen a objetos que yacen en clases distintas. Mientras menor sea la confusión más útil será para diferenciar las clases involucradas en un proceso de clasificación [15].

\section{Testor y Testor Típico. Concepto extendido}

Dado un conjunto de columnas $\tau$ de una tabla $\mathrm{T}$ formada por descripciones de objetos pertenecientes a las clases T0 y T1, si al eliminar de T todas las columnas, excepto las de $\tau$, no existe fila alguna en T0 semejante (de acuerdo a algún criterio de comparación) con alguna fila de $\mathrm{T} 1$, entonces $\tau$ se denomina testor de la tabla $\mathrm{T}$ para las clases $\mathrm{T} 0$ y T1. Un testor se llama típico (irreducible) si al eliminar cualquiera de sus columnas deja de ser un testor para las clases. Si en la tabla T existen objetos semejantes, pertenecientes a clases diferentes, entonces no se podrán obtener testores, según lo planteado.

En problemas reales de clasificación es difícil encontrar descripciones originales de las clases en matrices sin solapamiento (clases disjuntas), es decir que ningún objeto de una clase sea semejante a algún otro ubicado en otra clase. La definición extendida de testor plantea entonces que, dado un conjunto de columnas $\tau$ de una tabla $\mathrm{T}$, que puede tener o no objetos semejantes, si después de eliminar de T todas las columnas, excepto las de $\tau$, no aparecen nuevas sub-descripciones semejantes en clases diferentes, entonces $\tau$ se denomina testor de la tabla T [15].

Determinar todos los testores típicos de un problema generalmente requiere de un tiempo de procesamiento prolongado, que depende significativamente del número de rasgos utilizados y también del número de objetos involucrados en la descripción. Existen varios algoritmos para el cálculo de los testores típicos que disminuyen apreciablemente el tiempo de cómputo. Descubrir estrategias que logren cada vez un mejor rendimiento en el descubrimiento de los testores típicos puede ser vital para encontrar la solución óptima de un problema dado de reconocimiento de patrones. LEX, CT-EXT y Fast-BR son algunos de los algoritmos más destacados actualmente ante tales fines [16-18].

\section{Relevancia de los rasgos}

Los testores ofrecen una clara visión de que existen rasgos que aportan mayor información que otros en la clasificación de los objetos. A continuación, 
se describen las medidas de relevancia de un rasgo utilizadas en este trabajo. La relevancia de un rasgo puede determinarse a partir de la cantidad de testores típicos de los que forma parte, atendiendo a la siguiente expresión:

$$
P(x)=\frac{\omega(x)}{\omega}
$$

donde $\omega$ es el número total de testores típicos y $\omega(x)$ es el número de testores típicos donde aparece el rasgo $x$. Según esta medida, un rasgo es más importante en la medida que aparece en una mayor cantidad de testores típicos. Como una medida de importancia complementaria aparece la relevancia longitudinal de un rasgo:

$$
L(x)=\frac{\sum t \in \omega(x) \frac{1}{t}}{\omega(x)}
$$

donde $t$ representa la longitud de cada testor típico donde aparece el rasgo $x$ y $\omega(x)$ representa la cantidad de testores típicos que lo contienen.

Finalmente se expresa la relevancia de un rasgo a partir de una función que considera las medidas anteriores.

$$
\rho(x)=\alpha P(x)+\beta L(x)
$$

donde los parámetros $\alpha$ y $\beta$ son parámetros de ponderación de la influencia en la relevancia de los factores $P x$ y $L x$, respectivamente [15].

\section{Datos y metodología empleada}

El trabajo se realizó sobre un conjunto de datos proporcionados por especialistas de diagnóstico de una termoeléctrica a partir de las mediciones de las vibraciones de una chumacera durante tres condiciones de fallo diferentes: roturas de babbitt inferior (BI), holguras excesivas $(\mathrm{H})$ y roturas de babbitt inferior y holguras excesivas simultáneamente (BIH). Los datos derivados de las mediciones de las vibraciones en las direcciones horizontal y vertical se trabajaron de manera independiente debido a que los especialistas de diagnóstico sugieren que los fallos se manifiestan de forma diferente en una y otra dirección de medición. Cada conjunto está formado por 215 objetos pertenecientes a la clase BI, 354 objetos pertenecientes a la clase $\mathrm{H}$ y 553 objetos pertenecientes a la clase $\mathrm{BIH}$.

Los rasgos utilizados para describir cada uno de los objetos se determinaron a partir de la opinión de los expertos de diagnóstico sobre los registros de vibraciones. Por un lado, de los espectros de amplitud de las vibraciones se obtuvieron 18 variables de naturaleza numérica: 8 variables son los valores de amplitud de los armónicos (1X a 8X), 7 variables son los valores de amplitud de los interarmónicos presentes, y 3 variables corresponden a frecuencias sub-síncronas. Por otro lado, se obtuvieron 18 variables de naturaleza cualitativa: 1 variable identifica el nombre de la componente predominante en el espectro sin considerar la síncrona (Pred) y 17 variables contienen la relación de las amplitudes de los rasgos espectrales respecto a la amplitud de la componente síncrona (O2 a O18). "Pred" es una variable nominal y los 17 rasgos restantes son ordinales.

A través del cálculo de la confusión entre las clases fue verificada la efectividad de la aplicación de diferentes criterios de comparación y se logró la reducción del conjunto inicial de rasgos. No solo se realizó el cálculo de la confusión entre las clases, es decir, entre BI y $\mathrm{H}$, entre $\mathrm{H}$ y BIH, y entre BI y $\mathrm{BIH}$, sino que además se determinó la confusión entre los objetos de una misma clase, es decir, la confusión de BI, la confusión de $\mathrm{H}$ y la confusión de BIH. La confusión de un rasgo (o grupo de rasgos) entre clases se determinó a través de la definición aportada en la Sección 2,2. La confusión de un rasgo (o grupo de rasgos) para una misma clase se determinó en este trabajo como la cantidad de ocasiones en que los valores de un rasgo (o grupo de rasgos) fueron encontrados no semejantes. La semejanza se determina de acuerdo a un determinado criterio de comparación. El procedimiento de reducción del conjunto de rasgos, así como de selección del criterio de comparación a utilizar para cada rasgo, se basó en la determinación de aquellos rasgos con los que no se sobrepasó un 35\% de confusión, tanto para la confusión entre las clases, como para las confusiones propias de cada clase. Cuando varios criterios de comparación no sobrepasan el 35\% de confusión para el mismo rasgo, se selecciona el criterio de comparación que arroja menor porciento de confusión. El cálculo de la confusión siguiendo la 
metodología antes descrita se llevó a cabo empleando los criterios de comparación CC2, CC 3 y CC4, para los rasgos numéricos, y los criterios de comparación $\mathrm{CC} 1$ y CC2c para los rasgos ordinales y nominales.

Como la mayor parte de los criterios empleados necesitan de la definición de umbrales, intervalos, o conjuntos de datos que sustenten el cálculo de la semejanza, nuevamente la opinión de los especialistas juega un rol importante en su determinación. De esta forma, para el trabajo con CC2 se definieron 5 grupos de intervalos, para el trabajo con CC 3 se definieron 7 umbrales y para el trabajo con $\mathrm{CC} 2 \mathrm{c}$ se definieron 5 grupos de conjuntos de datos. Para indicar semejanza a la salida de los criterios CC4 se definieron 8 umbrales. Los experimentos se desarrollaron atendiendo todas las posibilidades mencionadas, identificando los parámetros que aportan mejores resultados con cada criterio.

Los resultados de la confusión permitieron el cálculo de los testores típicos y la importancia informacional de los rasgos presentes en ellos, aplicando las definiciones dadas en los epígrafes 2,3 y 2,4 , respectivamente.

\section{RESULTADOS}

A continuación, se exponen los resultados del trabajo, comenzando por el cálculo de la confusión. En las Tablas 1 y 2 se muestran los mejores resultados de los cálculos de confusión, donde aparecen 3 columnas, una para cada par de clases posibles, mientras que cada fila representa uno de los rasgos sobrevivientes del procedimiento de reducción de rasgos. Entre paréntesis aparecen los criterios de comparación con que se obtuvieron los mejores resultados.

De un total de 18 rasgos numéricos presentes en la descripción de cada objeto las variables se redujeron a solo 4 rasgos, para un $22,2 \%$, tanto para el conjunto de datos de las vibraciones de la dirección horizontal (Tabla 1), como de la dirección vertical (Tabla 2). De los 3 criterios de comparación empleados para comparar los rasgos numéricos, el más efectivo es el CC3 ya que a través de este criterio se obtienen los más bajos porcientos de confusión de modo general.

En el caso de los 18 rasgos cualitativos quedaron 7 variables $(38,9 \%)$ para el conjunto de datos correspondiente a las vibraciones de la dirección horizontal (Tabla 1) y 4 variables $(22,2 \%)$ para el
Tabla 1. Mejores resultados de confusión. Dirección horizontal.

\begin{tabular}{|c|c|c|c|}
\hline \multicolumn{4}{|c|}{ Confusión (\%) (dirección horizontal) } \\
\hline \multirow{2}{*}{ Rasgo } & \multicolumn{3}{|c|}{ Parejas de fallos } \\
\hline & BI-H & H-BIH & BI-BIH \\
\hline $1 \mathrm{X}$ & - & - & $29,49_{(\mathrm{CC} 2)}$ \\
\hline $3 X$ & $4,47_{(\mathrm{CC} 3)}$ & $24,12_{(\mathrm{CC} 4)}$ & $29,94_{(\mathrm{CC} 3)}$ \\
\hline $4 \mathrm{X}$ & $9,13_{(\mathrm{CC} 3)}$ & $15,38_{(\mathrm{CC} 3)}$ & - \\
\hline $5 X$ & - & $15,98_{(\mathrm{CC} 3)}$ & $27,95_{(\mathrm{CC} 4)}$ \\
\hline $\mathrm{O} 3$ & $6,2_{(\mathrm{CC} 2 \mathrm{c})}$ & $4,5_{(\mathrm{CC} 2 \mathrm{c})}$ & $7,1_{(\mathrm{CC} 2 \mathrm{c})}$ \\
\hline $\mathrm{O} 4$ & $18,58_{(\mathrm{CC} 2 \mathrm{c})}$ & $6,98_{(\mathrm{CC} 2 \mathrm{c})}$ & $32,54_{(\mathrm{CC} 1)}$ \\
\hline O5 & - & - & $34,63_{(\mathrm{CC} 1)}$ \\
\hline O7 & - & $6,12_{(\mathrm{CC} 2 \mathrm{c})}$ & - \\
\hline $\mathrm{O} 8$ & - & $25,37_{(\mathrm{CC} 2 \mathrm{c})}$ & - \\
\hline O16 & $30,03_{(\mathrm{CC} 1)}$ & $17,47_{(\mathrm{CC} 1)}$ & - \\
\hline Pred & - & $16,48_{(\mathrm{CC} 1)}$ & $27,47_{(\mathrm{CCl})}$ \\
\hline
\end{tabular}

Tabla 2. Mejores resultados de confusión. Dirección vertical.

\begin{tabular}{|c|c|c|c|}
\hline \multicolumn{4}{|c|}{ Confusión (\%) (dirección vertical) } \\
\hline \multirow{2}{*}{ Rasgo } & \multicolumn{3}{|c|}{ Parejas de fallos } \\
\cline { 2 - 4 } & BI-H & H-BIH & BI-BIH \\
\hline $2 \mathrm{X}$ & - & - & $17,96_{(\mathrm{CC} 3)}$ \\
\hline $3 \mathrm{X}$ & $11,3_{\mathrm{CC} 3)}$ & $24,82_{(\mathrm{CC} 3)}$ & - \\
\hline $4 \mathrm{X}$ & $3,24_{(\mathrm{CC} 3)}$ & $16,25_{(\mathrm{CC} 3)}$ & $13,97_{(\mathrm{CC} 3)}$ \\
\hline $8 \mathrm{X}$ & $12,07_{(\mathrm{CC} 3)}$ & $15,21_{(\mathrm{CC} 3)}$ & - \\
\hline O4 & - & $26,05_{(\mathrm{CC} 1)}$ & - \\
\hline O5 & $17,89_{(\mathrm{CC} 1)}$ & - & - \\
\hline O8 & $13,28_{(\mathrm{CC} 1)}$ & $15,11_{(\mathrm{CC} 1)}$ & - \\
\hline Pred & - & $30,01_{(\mathrm{CC} 2 \mathrm{c})}$ & $32,67_{(\mathrm{CC} 1)}$ \\
\hline
\end{tabular}

conjunto de datos correspondiente a las vibraciones de la dirección vertical (Tabla 2).

Los porcientos de confusión logrados con el conjunto de rasgos numéricos de las vibraciones de la dirección horizontal fueron significativamente mejorados por los alcanzados trabajando con el conjunto de datos cualitativos. Los mejores resultados de confusión se alcanzan con el criterio CC2c, superando los resultados alcanzados con $\mathrm{CC} 3$ con los rasgos numéricos.

También con las variables cualitativas se obtienen menores porcientos de confusión para diferenciar 
la clase BIH de las clases BI y $\mathrm{H}$ en la dirección horizontal, como aparece en la Tabla 1, y para diferenciar las clases $\mathrm{H}$ y BIH en la dirección vertical (Tabla 2).

En las Tablas 3 y 4 aparecen los criterios de comparación seleccionados para cada rasgo, así como los parámetros de cada criterio que permitieron alcanzar los mejores resultados. Se aprecia que para los rasgos numéricos $3 \mathrm{X}$ y $4 \mathrm{X}$ el criterio de comparación más eficiente fue el CC3, con el parámetro umbral (u) igual a 0,5 para ambas direcciones de medición de las vibraciones. Este criterio también resultó el mejor para los rasgos $5 \mathrm{X}$ en las vibraciones de la dirección horizontal y el rasgo $2 \mathrm{X}$ en la dirección vertical (en ambos casos con $\mathrm{u}=0,7$ ), así como para el rasgo $8 \mathrm{X}$ en las vibraciones de la dirección vertical, esta vez con $\mathrm{u}=0,25$. En los rasgos cualitativos, el criterio $\mathrm{CC} 2 \mathrm{c}$, con parámetro conjunto c5, resultó mejor

Tabla 3. Criterios de comparación. Dirección horizontal.

\begin{tabular}{|c|c|c|}
\hline Rasgo & Criterios & Parámetros \\
\hline $1 \mathrm{X}$ & $\mathrm{CC} 2$ & $\mathrm{a} 2$ \\
\hline $3 \mathrm{X}$ & $\mathrm{CC} 3$ & $\mathrm{u}=0,5$ \\
\hline $4 \mathrm{X}$ & $\mathrm{CC} 3$ & $\mathrm{u}=0,5$ \\
\hline $5 \mathrm{X}$ & $\mathrm{CC} 3$ & $\mathrm{u}=0,7$ \\
\hline $\mathrm{O} 3$ & $\mathrm{CC} 2 \mathrm{c}$ & $\mathrm{c} 5$ \\
\hline $\mathrm{O} 4$ & $\mathrm{CC} 2 \mathrm{c}$ & $\mathrm{c} 4$ \\
\hline $\mathrm{O} 5$ & $\mathrm{CC} 1$ & - \\
\hline $\mathrm{O} 7$ & $\mathrm{CC} 2 \mathrm{c}$ & $\mathrm{c} 2$ \\
\hline $\mathrm{O} 8$ & $\mathrm{CC} 2 \mathrm{c}$ & $\mathrm{c} 5$ \\
\hline O16 & $\mathrm{CC} 1$ & - \\
\hline Pred & $\mathrm{CC} 1$ & - \\
\hline
\end{tabular}

Tabla 4. Criterios de comparación. Dirección vertical.

\begin{tabular}{|c|c|c|}
\hline Rasgo & Criterios & Parámetros \\
\hline $2 \mathrm{X}$ & $\mathrm{CC} 3$ & $\mathrm{u}=0,7$ \\
\hline $3 \mathrm{X}$ & $\mathrm{CC} 3$ & $\mathrm{u}=0,5$ \\
\hline $4 \mathrm{X}$ & $\mathrm{CC} 3$ & $\mathrm{u}=0,5$ \\
\hline $8 \mathrm{X}$ & $\mathrm{CC} 3$ & $\mathrm{u}=0,25$ \\
\hline O4 & $\mathrm{CC} 1$ & - \\
\hline O5 & $\mathrm{CC} 1$ & $-\mathrm{q}$ \\
\hline O8 & $\mathrm{CC} 1$ & - \\
\hline Pred & $\mathrm{CC} 2 \mathrm{c}$ & $\mathrm{c} 2$ \\
\hline
\end{tabular}

para las variables $\mathrm{O} 3$ y $\mathrm{O} 8$ en las vibraciones de la dirección horizontal. También este criterio fue mejor para el rasgo $\mathrm{O} 7$ de las vibraciones de la dirección horizontal y para el rasgo Pred de las vibraciones en la dirección vertical, en ambos casos con conjunto c2. Por último, este criterio también resultó más eficiente con conjunto c4 en el rasgo $\mathrm{O} 4$ de las vibraciones en la dirección horizontal. El criterio $\mathrm{CC} 1$, que no necesita de parámetros, se destacó en las comparaciones de las variables O5 y Pred de las vibraciones en la dirección horizontal, así como en los rasgos O4, $\mathrm{O} 5$ y $\mathrm{O} 8$ de las vibraciones en la dirección vertical. Los criterios $\mathrm{CC} 2$ con parámetro intervalo a2 y CC1, fueron los más eficientes para los rasgos $1 \mathrm{X}$ y $\mathrm{O} 16$ de las descripciones numéricas y cualitativas, respectivamente, de las vibraciones en la dirección horizontal.

En las Tablas 5 y 6 se muestran los testores típicos que se obtuvieron para diferenciar las clases correspondientes, tanto para el conjunto de datos de vibraciones de la dirección horizontal (Tabla 5) como para las vibraciones de la dirección vertical (Tabla 6). Se aprecia que las variables cualitativas que se consideran tienen una participación decisiva en la diferenciación de cada una de estas clases, pues forman parte de todos los testores encontrados. En las vibraciones de la dirección horizontal las variables cualitativas son mayoría $(66,7 \%)$ entre los rasgos que conforman los testores. Mientras en las vibraciones de la dirección vertical todos los testores están formados por una variable solamente, y es nominal. En las vibraciones de la dirección horizontal se eliminan los rasgos $5 \mathrm{X}$ y $\mathrm{O} 8$ porque no aparecen en los testores típicos. También se eliminan los rasgos $2 \mathrm{X}, 3 \mathrm{X}, 4 \mathrm{X}$, $8 \mathrm{X}, \mathrm{O} 4, \mathrm{O} 5$ y $\mathrm{O} 8$ del conjunto de datos de vibraciones de la dirección vertical, ya que no aparecen en el único testor típico de estas clases.

El cálculo del peso informacional de cada uno de los rasgos que conforman los testores típicos se realizó de acuerdo con la ecuación (8), obteniéndose los

Tabla 5. Testores típicos. Dirección horizontal.

\begin{tabular}{|c|c|c|c|c|c|c|c|}
\hline \multirow{2}{*}{ Fallo } & \multicolumn{7}{|c|}{ Testores Típicos } \\
\hline \multirow{2}{*}{ BI-H } & $1 \mathrm{X}$ & O3 & O4 & O5 & O16 & - & - \\
\cline { 2 - 8 } & 1X & $4 \mathrm{X}$ & O3 & O4 & O16 & - & - \\
\hline \multirow{2}{*}{ H-BIH } & O3 & - & - & - & - & - & - \\
\cline { 2 - 8 } & $3 \mathrm{X}$ & - & - & - & - & - & - \\
\hline \multirow{2}{*}{ BI-BIH } & $1 \mathrm{X}$ & $3 \mathrm{X}$ & $4 \mathrm{X}$ & $\mathrm{O} 4$ & $\mathrm{O} 5$ & $\mathrm{O}$ & Pred \\
\hline
\end{tabular}


Tabla 6. Testores típicos. Dirección vertical.

\begin{tabular}{|c|c|}
\hline Fallo & Testor \\
\hline BI-H & Pred \\
\hline H-BIH & Pred \\
\hline BI-BIH & Pred \\
\hline
\end{tabular}

resultados que se muestran en la Tabla 7. Entre los rasgos de los testores típicos con mayores índices de importancia abundan los rasgos cualitativos, pues 3 de los 5 rasgos con un índice de importancia mayor de 0,25 son cualitativos. El rasgo con mayor índice de importancia es el rasgo nominal Pred, pues es el único testor encontrado para diferenciar las 3 clases a partir de las vibraciones de la dirección vertical y aparece en 4 de los 8 testores típicos encontrados. Se corrobora que los largos 5X y O8 deben eliminarse porque tienen índice de importancia 0 ya que no aparecen en ninguno de los testores típicos encontrados. En resumen, 6 de los 9 rasgos sobrevivientes son cualitativos para un $66,6 \%$.

\section{CONCLUSIONES}

Este trabajo constituye la aplicación del RLCP a la identificación de los rasgos más importantes que describen fallos de chumaceras, empleando por primera vez datos obtenidos de los fallos registrados durante la explotación en un entorno industrial.

Este trabajo representa por primera vez la incorporación de conocimiento experto expresado en variables no numéricas a la selección de los rasgos más importantes para el diagnóstico de fallos de chumaceras.

Tabla 7. Relevancia de los rasgos.

\begin{tabular}{|c|l|l|l|}
\hline Rasgo & $\boldsymbol{P} \boldsymbol{x}$ & $\boldsymbol{L} \boldsymbol{x}$ & $\boldsymbol{\rho} \boldsymbol{x}$ \\
\hline Pred & 0,5 & 0,7857 & 0,6428 \\
\hline O3 & 0,375 & 0,47 & 0,4525 \\
\hline $3 \mathrm{X}$ & 0,25 & 0,57 & 0,41 \\
\hline O4 & 0,375 & 0,18 & 0,2775 \\
\hline 1X & 0,375 & 0,18 & 0,2775 \\
\hline O16 & 0,25 & 0,2 & 0,225 \\
\hline 4X & 0,25 & 0,17 & 0,21 \\
\hline O5 & 0,25 & 0,17 & 0,21 \\
\hline O7 & 0,125 & 0,14 & 0,1325 \\
\hline 5X & 0 & 0 & 0 \\
\hline O8 & 0 & 0 & 0 \\
\hline
\end{tabular}

La metodología empleada permitió identificar las variables más importantes para describir los fallos tratados, reduciendo significativamente el conjunto inicial de 36 variables consideradas a 11 y a 9 variables seleccionadas en la dirección de medición horizontal y a 1 variable en la dirección vertical.

Los resultados alcanzados mostraron que la relevancia de los rasgos cualitativos que se incorporaron a la descripción de los fallos es superior a la de los rasgos numéricos.

\section{AGRADECIMIENTOS}

A los especialistas de diagnóstico Julio González Martínez, Yuritza Cruz Guzmán, Jorge C. Arce Miranda y María Antonia Téllez.

\section{REFERENCIAS}

[1] I. El-Thalji and E. Jantunen. "A summary of fault modelling and predictive health monitoring of rolling element bearings". Mechanical Systems and Signal Processing. Vol. 60-61, pp. 252-272. 2015. ISSN 0888-3270.

[2] I. Khelf, L. Laouar, A.M. Bouchelaghem, D. Rémond and S. Saad. "Adaptive fault diagnosis in rotating machines using indicators selection". Mechanical Systems and Signal Processing. Vol. 40, Issue 2, pp. 452-468. 2013. ISSN 0888-3270.

[3] Y. Lei, J. Lin, Z. He and M.J. Zuo. "A review on empirical mode decomposition in fault diagnosis of rotating machinery". Mechanical Systems and Signal Processing. Vol. 35, Issue 1-2, pp. 108-126. 2013. ISSN 0888-3270.

[4] J. Pino Gómez, F.E. Hernández Montero, M.E. Montesinos Otero, M.A. Tellez, J. Gonzalez Martínez, Y. Cruz Gúzman and J.C. Arce Miranda. "Importancia para el mantenimiento de elementos mecánicos y fallos en turbinas de vapor. Análisis de históricos". Revista de Ingeniería Energética. Vol. XXXVIII N ${ }^{\circ} 2$, pp. 106-114. 2017. ISSN: 1815-5901.

[5] A. Bilošová and J. Biloš. "Vibrations Diagnostics". Ostrava: European Social Fund (ESF). 2012. DOI: CZ.1.07/2,2.00/15.0132.

[6] L.A. Branagan. "Survey of Damage Investigation of Babbitted Industrial 
Bearings". Lubricants, Issue 3, pp. 91-112. 2015. ISSN: 2075-4442.

[7] J. Gómez-Mancilla, M.A. Castillo-Ginori and A. Marín-Herrera. "A Turbocompressor Operating with Severe Misaligned Bearing Problems". In Procs. ASME- France, Workshop: Bearings Under Severe Operating Conditions, EDF/LMS Poitiers. Francia. 2002.

[8] J.C. Gomez-Mancilla, V. Nosov and G. SilvaNavarro. "Rotor-Bearing System Stability Performance Comparing Hybrid versus Conventional Bearings". International Journal of Rotating Machinery, Issue 1, pp. 16-22. 2005. ISSN: 1023-621X.

[9] K.M. Saridakis, P.G. Nikolakopoulos, C.A. Papadopoulos and A.J. Dentsoras. "Fault Diagnosis of Journal Bearings Based on Artificial Neural Networks and Measurements of Bearing Performance Characteristics". in Proceedings of the Ninth International Conference on Computational Structures Technology. Stirlingshire, UK. 2008. DOI: 10.4203/ccp.88.118.

[10] J. Byungchul. "Statistical Approach to Diagnostic Rules for Various Malfunctions of Journal Bearing System Using Fisher Discriminant Analysis". In European Conference of the prognostics and health management society. 2014. URL: http:// www.phmsociety.org

[11] T. Narendiranath, T. Manvel and T. Lakshmanan. "High Frequency Acceleration Envelope Power Spectrum for Fault Diagnosis on Journal Bearing using DEWESOFT". Research Journal of Applied Sciences, Engineering and Technology. Vol. 8, Issue 10, pp. 1225-1238. 2014. DOI: 10.19026/ rjaset.8.1088.

[12] A. Moosavian. "Comparison of two classifiers; $\mathrm{K}$-nearest neighbor and artificial neural network, for fault diagnosis on a main engine journal-bearing". Shock and Vibration. Vol. 20, Issue 2, pp. 263-272. 2013. DOI: $10.1155 / 2013 / 360236$.

[13] K.M. Saridakis, P.G. Nikolakopoulos, C.A. Papadopoulos and A.J. Dentsoras. "Identification of wear and misalignment on journal bearings using artificial neural networks". Journal of Engineering Tribology, Issue 1. 2012.

[14] J. Ruiz-Shulcloper. "Pattern Recognition with Mixed and Incomplete Data". Pattern Recognition and Image Analysis. Vol. 18, Issue 4, pp. 563-576. 2008. ISSN: 1054-6618.

[15] J. Ruiz-Shulcloper. "Logical Combinatorial Pattern Recognition: A Review". Recent Research Developments in Pattern Recognition. Vol. 3,pp. 133-176. 2002. ISBN: 81-7895-050-2.

[16] A. Lias Rodríguez and G. Sanchez Diaz. "An algorithm for computing typical testors based on elimination of gaps and reduction of columns". International Journal of Pattern Recognition and Artificial Intelligence. Vol. 27, Issue 8, pp. 1350022-(1-18). 2013. DOI: $10.1142 / \mathrm{S} 0218001413500225$.

[17] G. Sanchez Diaz and M. Lazo-Cortés. "CTEXT: An Algorithm for Computing Typical Testor Set”. LNCS. Vol. 4756, pp. 06-14. 2007. Springer-Verlag Berlin Heidelberg.

[18] Y. Santiesteban Alganza and A. Pons Porrata. "LEX: A new algorithm for calculating typical testors". Revista Ciencias Matematicas. Vol. $21 \mathrm{~N}^{\mathrm{o}}$ 1, pp. 85-95. 2003. 